\title{
Reflets
}

Revue ontaroise d'intervention sociale et communautaire

\section{À l'aide des femmes francophones qui luttent contre les agressions à caractère sexuel}

\section{Gaëtane Pharand}

Volume 2, numéro 1, printemps 1996

Contrer la violence subie par les femmes et les enfants en milieu

familial

URI : https://id.erudit.org/iderudit/026108ar

DOI : https://doi.org/10.7202/026108ar

Aller au sommaire du numéro

Éditeur(s)

Reflets : Revue ontaroise d'intervention sociale et communautaire

ISSN

1203-4576 (imprimé)

1712-8498 (numérique)

Découvrir la revue

Citer cet article

Pharand, G. (1996). À l'aide des femmes francophones qui luttent contre les agressions à caractère sexuel. Reflets, 2(1), 109-115.

https://doi.org/10.7202/026108ar

Tous droits réservés (C) Reflets : Revue ontaroise d'intervention sociale et communautaire, 1996
Ce document est protégé par la loi sur le droit d'auteur. L'utilisation des services d'Érudit (y compris la reproduction) est assujettie à sa politique d'utilisation que vous pouvez consulter en ligne.

https://apropos.erudit.org/fr/usagers/politique-dutilisation/ 


\section{À l'aide des femmes francophones qui luttent contre les agressions à caractère sexuel}

\section{$\mathbf{G}$ aëtane $\mathbf{P}$ harand}

A gente de coordination $C$ entreV ictoria pour femmes Sudbury

\section{Historique}

En 1992, dansle cadre de l'implantation de la loi 8 sur les services en français, le ministère du Solliciteur général et des Services correctionnels commandait une étude sur les besoins desfemmes francophones victimesd'agressionsà caractère sexuel dans la région de Sudbury. Cette étude, effectuée par le Sexual A ssault C risis $C$ entre de Sudbury, montre que les services en français sont quasi inexistants. Les participantes à l'étude soulignent, entre autres, l'urgence de la mise sur pied d'une ligne de crise pour répondre aux besoins des femmes francophones. Invité à prendre connaissance des résultats de cette recherche, un groupe de femmes francophones intervenant dans le dossier de la violence faite aux femmes, se réunissait pour établir la base de ce qui est devenu aujourd'hui le C entreV ictoria.

\section{Mandat}

La mission première du C entreVictoria est d'offrir des services en français pour les femmes âgées de 16 ans et plus dans la région 
de Sudbury et qui ont subi une ou plusieurs formes d'agressions à caractère sexuel (viol, inceste, attouchements, harcèlement sexuel). $D$ ès ses débuts, les membres et les bénévoles du $C$ entre Victoria ont insisté sur le besoin de créer non pas une agence de «counselling» mais plutôt un lieu sécuritaire et chaleureux, où il est possible d'obtenir du soutien, et où les rapports inégaux de pouvoir entre les personnes ne doivent pas exister. II s'agit davantage d'accompagner les femmes dans leur cheminement personnel de victime, de survivante à vivante. $D$ e plus, les femmes qui utilisent les services ne sont pas des clientes, mais des participantes au processus de cheminement axé sur leurs besoins, expériences et réalités. C'est donc dans cette optique, et à la demande des femmes, que le $\mathrm{C}$ entreV ictoria a établi la ligne de crise.

Le 8 mars 1995, lors de la Journée internationale des femmes, le CentreV ictoria inaugurait son service. La ligne de crise a été mise en opération. Pendant les trois premiers mois, elle n'a opéré que 13,5 heures par jour. À la mi-juin, ce service passait à 24 heures, 7 jours par semaine. Le délai avant de passer à un plein service a permis au C entre Victoria de s'ajuster à la réalité d'un service très exigeant au niveau de la formation et de l'encadrement technique et psychologique, tout en tenant compte des engagements professionnels et personnels de ses bénévoles.

Le $\mathrm{C}$ entre Victoria a également été mandaté pour faire des interventions communautaires de sensibilisation, d'éducation et de prévention. II offre aussi des services d'accompagnement individuel (à la police, au tribunal, à l'hôpital, etc.), de soutien individuel et de groupe, d'aiguillage aux ressources et aux divers services disponibles dansla communauté, et de défense desdroits.

\section{Marrainage et partenariats}

D ans sa recherche d'une identité, et pour refléter le vécu et la réalité des femmes agressées sexuellement, les membresont choisi 
de donner au centre le nom d'une survivante. $\mathrm{N}$ otre marraine estV ictoria M onkman, activiste féministe dans le domaine de la violence faite aux femmes. Elle a oeuvré àT immins dans le nord de l'O ntario, dans un centre semblable. Elle a fait des études en sciences politiques, histoire, pédagogie et études de la femme. Victoria M onkman incarne notre philosophie et notre orientation, car elle représente le cheminement positif de victime à survivante. C'est une vivante parmi beaucoup d'autres, et elle demeure une source d'inspiration pour les travailleuses et bénévoles du C entreV ictoria.

Pour permettre le ressourcement, le réseautage et le soutien mutuel, le C entreV ictoria s'est asso cié à des organismes qui partagent ses préoccupations. D epuis février 1995, trois organismes se sont regroupés pour partager bureaux, équipements, projets et activités, et pour consolider certains services. C esorganismes sont le $\mathrm{C}$ entre $\mathrm{V}$ ictoria, la Fédération des femmes canadiennesfrançaises de l'O ntario (FFCF de l'O ntario) et le Collectif desfemmes francophones du N ord-Est ontarien. D'ores et déjà, grâce à cette association, nous avons créé quelques emplois, un centre de ressources et un lieu de rencontre pour les femmes francophones. G râce à la mise en commun des ressources, nous possédons plus de 2000 titres (vidéos, ateliers, articles, etc.) sur des questions touchant la réalité des femmes (économie, santé, sexualité, abus, etc.). C es ressources sont à la disposition de la communauté.

En vue de représenter et défendre les droits des femmes francophones nord- ontariennes, le $\mathrm{C}$ entreV ictoria est aussi membre de deux groupes provinciaux : I'A cion ontarienne contre la violence faite aux femmes et la Table féministe francophone de concertation provinciale.

\section{Réalités et obstacles}

En O ntario français, il n'existait pas de services d'urgence pour répondre aux besoins des femmes victimes d'agressions à caractère sexuel. O n ne trouvait pas non plus d'outils de formation, 
ni de ressources en français adaptés aux besoins des femmes francophones. À ces lacunes flagrantes, comblées par le Centre Victoria, s'est ajoutée l'obligation, imposée par le financement provincial, d'offrir des services assurés surtout par des bénévoles. $C^{\prime}$ 'est donc dire qu'il n'est pas suffisant d'avoir à composer avec nos réalités en tant que femmes et francophones, il faut, de plus, donner gratuitement temps et énergie à toute une panoplie d'activités. D es tâches comme le recrutement, la formation, l'encadrement et la reconnaissance des bénévoles, l'offre des services directs, la promotion, la prévention et la sensibilisation, la recherche de financement et la gestion ne peuvent être accomplies seulement par deux intervenantes. Les bénévoles jouent ainsi un rôle indispensable en acceptant certaines de ces responsabilités, afin d'assurer la croissance et la viabilité du centre. Le C entre Victoria est toutefois privilégié parce qu'il bénéficie d'un noyau solide de femmes de la communauté. C elles-ci sont dévouées, et surtout convaincues de la nécessité des services communautaires en français pour victimes d'agressions à caractère sexuel.

G râce au travail bénévole des femmes, il existe en 0 ntario, à la fin de 1995, trois centres francophones de lutte contre les agressions à caractère sexuel ( 0 ttawa, Sudbury, Toronto), et des services en français offerts par des agences bilingues dans onze (11) localités (B arrie, C hatham-Kent, H amilton, Sarnia,W indsor, Cornwall, N orth Bay, N iagara, T immins, Pembroke- $R$ enfrew, Sault-Ste-M arie). Seule la communauté francophone du N ordouest demeure sans service d'urgence pour les francophones victimes d'agressions à caractère sexuel.

Les services d'aide et de lutte aux agressionsà caractère sexuel qui existent sont subventionnés par le ministère provincial du Solliciteur général et des Services correctionnels. Les subventions sont accordées en fonction de la population francophone de chacune des localités. Au recensement de 1991, la municipalité régionale de Sudbury comptait une population totale de 160200 habitants, dont 43310 francophones. Près de 30610 personnes disent parler le français à la maison et 60055 personnes connaissent les deux langues officielles. La population francophone donne donc droit à un financement annuel maximum de 120000 \$. 
Pour son année financière d'octobre 1995 à septembre 1996, le C entreV ictoria s'attendait à recevoir ce maximum. C ependant, en raison de coupures budgétaires, tous les centres ont subi à l'automne 1995, une coupure de $4,8 \%$, rétroactive au premier avril 1995. À la rédaction de cet article, les centres et services en français ont reçu suffisamment de fonds pour opérer jusqu'à la fin mars 1996. L'intention du nouveau gouvernement $\mathrm{H}$ arris, face aux services aux victimes, déterminera la place qui sera accordée aux besoins des femmes francophones. Le financement précaire mis à part, il n'en reste pas moins que l'un des plus grands défis des services en français pour femmesfrancophones demeure celui d'amener lesfemmes à rompre le silence sur lesagressions sexuelles qu'elles ont vécues. Celui-ci s'est fait complice de leur honte, culpabilité, insécurités et peurs. Pour l'instant, mal gré les difficultés évidentes d'un personnel restreint et de la précarité du travail de bénévolat, le $\mathrm{C}$ entre Victoria continue à gérer son travail de façon indépendante en français.

\section{Réalisations}

Le C entreV ictoria n'existe officiellement que depuis un an. Sans compter toutes les heures de travail effectuées afin de mettre sur pied le centre (pendant près de 2 ans), du 8 mars au 30 septembre 1995, nous pouvons parler des réalisations suivantes:

- les membres et les bénévoles ont consacré plus de 600 heures à la formation;

- nous comptons 2478 heures de bénévolat pour répondre aux besoins de la ligne de crise, et aux rencontres et activités du centre;

- nous avons répondu à 61 appels de crise d'une durée variée, et offert des services directs à de nombreuses femmes (consultations d'urgence, consultations individuelles, appuis directs et autres interventions de soutien); 
- nous avons participé à 56 activités de sensibilisation ou de formation du public pour une assistance totale de 897 personnes;

- nous avons reçu et dirigé 34 appels d'information et d'assistance pratique (aiguillage aux ressources communautaires) et finalement,

- nous avonsparticipéà 9 rencontres de développement communautaire (comités locaux et collaboration entre organismes).

Pour protéger la santé des bénévoles et du personnel, et pour éviter de créer des attentes irréalisables auprès de notre population cible, le Centre Victoria a choisi, pour sa première année d'opération, de concentrer ses énergies sur son service de ligne de crise. Grâce à d'autres programmes de subventions, le $C$ entre $V$ ictoria compte toutefois réussir à combler certains besoins pressants. D'ici avril 1996, nous serons en possession d'une trousse de formation des bénévoles, composée d'une quinzaine de fiches thématiques permettant d'offrir près de 50 heures de formation. Un projet de formation d'animatrices pour la constitution de groupes de soutien comblera aussi un autre besoin. Par ailleurs, nos plans à long terme visent la consolidation de nos effectifs de bénévolat et de personnel, la promotion des services et la poursuite des partenariats avec d'autres agences ou organismes communautaires.

\section{Conclusion}

Avec l'ouverture du C entre Victoria, les femmes francophones de la région de Sudbury ont un service qui répond à leurs besoins. $M$ ais, «mettre fin à la violence faite aux filles et aux femmes suppose un changement radical des méthodes de distribution du pouvoir et des avoirs dans notre société. Le premier de ces changements repose sur la multiplication des choix qui seront offerts et accessibles à toutes les femmes de nos communautés francophones.» I| s'agit là de la raison d'être du CentreVictoria pour 
les femmes francophones qui luttent contre les agressions à caractère sexuel (Sudbury). Elles sont inscrites dans ce mouvement de changement. 